\title{
Validation of the instrumented evaluation of spatio-temporal gait parameters in patients with motor incomplete spinal cord injury
}

\author{
AI Pérez-Sanpablo ${ }^{1}$, J Quinzaños-Fresnedo ${ }^{2}$, R Loera-Cruz ${ }^{2}$, I Quiñones-Uriostegui ${ }^{1}$, G Rodriguez-Reyes ${ }^{3}$ \\ and R Pérez-Zavala ${ }^{2}$
}

STUDY DESIGN: Observational, descriptive, transversal.

OBJECTIVES: To evaluate the validity and reliability of spatio-temporal gait parameters measured by GaitRite in motor incomplete spinal cord-injured (SCl) patients.

SETTING: National Institute of Rehabilitation, Mexico city.

METHODS: 23 motor subacute and chronic incomplete SCI American Spinal Cord Injury Association Impairment Scale (AIS) D subjects were measured. The 10-meter walking test (10 MWT), 6-minute walking test (6 MWT), Walking Index for Spinal Cord Injury II (WISCI-II), Spinal Cord Independence Measure III (SCIM-III) and the GaitRite evaluation were carried out concurrently in order to determine validity. The $10 \mathrm{MWT}$ and GaitRite evaluation were performed at different occasions in order to determine test-retest reliability.

RESULTS: GaitRite offers a valid and reliable way to measure the mobility, symmetry and stability characteristics of gait SCl subjects. GaitRite precision and sensitivity is approximately three times better than clinical tests. Clinical tests cannot address the stability properties of gait. Subjects' higher gait velocity is related to more independence (SCIM-III), lower use of walking aids (WISCI-II), better performance in lower extremities motor score (LEMS) and better gait's mobility.

CONCLUSIONS: Spatio-temporal gait parameters measured by GaitRite are both valid and reliable. Further studies are necessary to establish sensitivity of the instrument.

Spinal Cord (2017) 55, 699-704; doi:10.1038/sc.2017.4; published online 28 February 2017

\section{INTRODUCTION}

Spinal cord injury (SCI) affects standing equilibrium, locomotion and voluntary movements. ${ }^{1,2}$ Global prevalence of traumatic SCI is from 236 to 4187 per million inhabitants, though missing prevalence data for major populations persist. ${ }^{3}$ More than $50 \%$ of individuals with SCI have motor incomplete lesions and more than $75 \%$ of these patients regain some form of ambulatory function. ${ }^{1,4}$ However, they are likely to walk with gait deficiencies like slow walking speed, abnormal step length, cadence and step symmetry that negatively impact walking efficiency. ${ }^{5}$ One of the goals in the rehabilitation of spinal cord injured individuals is to reduce these deficiencies. However, in order to assess the effect of those approaches sensitive walking measures are needed. Therefore, it is necessary to have valid, reliable and objective measures of functional walking performance. ${ }^{6}$

Several walking measures have been developed for and validated in SCI population: the Walking Index for Spinal Cord Injury II (WISCI-II), the $10 \mathrm{~m}$ walk test (10 MWT), and the 6 min walk test (6 MWT). All those tools have been recommended as outcome measures for gait in SCI. ${ }^{7,8}$ Also Spinal Cord Independence Measure (SCIM-III), which assesses independence in selfcare, mobility and sphincter management, has three specific items related to gait performance indoor and outdoor.

Validity refers to the ability of an instrument to assess what it is intended to measure $;{ }^{9}$ it encompasses construct and criterion validity. Concurrent validity, part of the criterion validity, is the degree to which a parameter is similar to other parameters to which it should be theoretically related. ${ }^{10}$ Construct validity refers to accumulated evidence that a test performs as expected when measuring an underlying trait or concept. There is no single best way to measure it, but convergent validity and discriminant validity are part of it. Convergent validity tests the degree of relation between measures that are theoretically related. Discriminant validity refers to the non-relation level of measures theoretically unrelated. Test-retest reliability indicates similarity of values measured in unchanged conditions several times. ${ }^{10}$ It has been quantified by intraclass correlation coefficient (ICC), the s.e.m. and the smallest real difference (SRD). ${ }^{11}$ The amount of change of a measure necessary to be detectable is estimated by the s.e.m., whereas the smallest change that represents a real (clinical) change is calculated by the SRD.

\footnotetext{
${ }^{1}$ Human Motion Analysis Laboratory, National Institute of Rehabilitation, Calzada México-Xochimilco No. 289, Col. Arenal de Guadalupe, Delegación Tlalpan, Mexico City, Mexico; ${ }^{2}$ Neurologic Rehabilitation Department at National Institute of Rehabilitation. Mexico City, Mexico and ${ }^{3}$ Prosthetics and Orthotics Laboratory, National Institute of Rehabilitation, Mexico City, Mexico

Correspondence: Dr J Quinzaños-Fresnedo, Instituto Nacional de Rehabilitación, Calzada México-Xochimilco No. 289, Col. Arenal de Guadalupe, Delegación Tlalpan, 14389 Mexico City, Mexico.

E-mail: jimenaqf@hotmail.com

Received 11 April 2016; revised 28 December 2016; accepted 5 January 2017; published online 28 February 2017
} 
All previous measures allow assessment of walking functionality. Two constructs can be considered to measure gait functionality: gait capacity and gait performance. ${ }^{6}$ Gait capacity evaluates the individuals' ability to walk in a standard environment. ${ }^{12}$ Gait performance measures the individuals' ability to walk in his/her usual environment. ${ }^{12}$ Indicators as velocity, frequency, time, space, symmetry and stability have been used to estimate gait capacity. Unfortunately, none of the previous scales (10 MWT, 6MWT, WISCI-II and SCIM-III) are capable to measure these indicators sensitively enough to evaluate potential interventions for the recovery of walking function. An indicator of gait functionality from a different construct is the use of gait facilitators such as canes or walkers. Other constructs less related to gait functionality are individuals' global independence and lower limb muscle capacity.

Technology-aided measures provide precise and quantitative data, but their use is affected by factors as cost, specialized equipment and time requirements. ${ }^{7}$ A feasible technology-aided alternative to measure gait capacity is a computerized walkway as the GaitRite walkway system (CIR Systems, Havertown, PA, USA). GAITRite is an instrumented mat composed of pressure sensors which detects footfalls. The mat is connected to a computer where a software calculates temporal and spatial gait parameters like step length, stride length, cadence, gait velocity, base of support, stance time and sway time. Its validity and reliability have been investigated in the elderly, ${ }^{13-15}$ in children with motor disabilities ${ }^{14}$ and knee replacement ${ }^{15}$ and in patients with Parkinson disease. ${ }^{16,17}$ Certainly three-dimensional optoelectronic motion capture systems such as VICON in combination with force platforms are considered to be the gold standard in evaluating accuracy of spatial-temporal measurements. ${ }^{18}$ Previous results showed that although significant differences have been found on few spatial and temporal measurements (such as step length, gait velocity and duration of gait phases), these differences are small $(1.5 \%$ on average $) .{ }^{18}$ However, there are no studies testing validity and reliability in subjects with SCI. Validity and reliability results cannot be extrapolated from other populations because SCI subjects have neuromuscular and mechanical particular impairments. ${ }^{7}$ Despite the lack of validation studies, GaitRite has been already used in report of cases and small series of cases ${ }^{17,19-22}$ because of the critical necessity of these measures.

Therefore, the overall aim of this study was to study metrological properties of GaitRite measurements by assessing the standard error of measurement, SRD, test-retest reliability, criterion validity and construct validity of the measurements which are particularly important within clinical practice for the evaluation of walking capacity of individuals with SCI. Consequently, we decided to compare GaitRite with tools already validated and of proven utility within clinical practice.

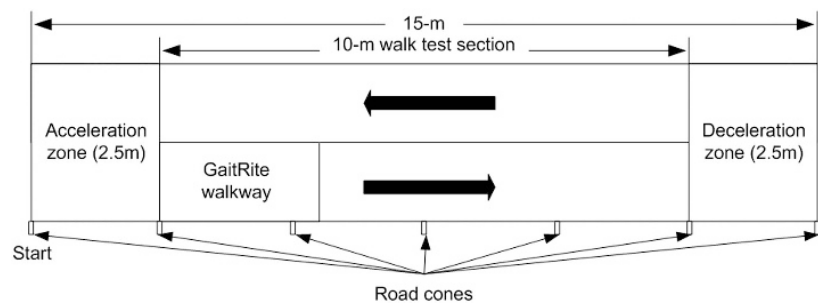

Figure 1 Walkway setup for concurrent application of 6 MWT, 10 MWT and GaitRite.

\section{METHODS}

Subjects younger than 60 years of age with subacute or chronic (more than 6 months of evolution) motor incomplete SCI American Spinal Injury Association Impairment Scale (AIS) D, with any neurological level and able to walk with or without aids were included. Subjects were excluded in the presence of cognitive deficits, cardiac or lung diseases which could limit patient's effort. Study was approved by local Ethical Committee, and written informed consent was obtained from all subjects. Demographic data and neurological status were recorded from all patients. Demographic data included the following: gender, age, time from onset and etiology of lesion. Neurological status was assessed by a physician specialized in neurological rehabilitation according to the International Standards for Neurological Classification of Spinal Cord Injury. Specialists also assessed lower-extremity motor score (LEMS), use of walking aids and walking level according to WISCI-II and SCIM-III. The use of walking aids was categorized in an ordinal scale starting from no aid, one cane, two canes and ending on walkers.

Subjects underwent concurrent application of $6 \mathrm{MWT}, 10 \mathrm{MWT}$ and GaitRite assessment in the same trial on the same walkway. 6 MWT was performed in a $15 \mathrm{~m}$ straight corridor with $180^{\circ}$ turns at edges, see Figure 1. Road cones were applied at the turn points and every $2.5 \mathrm{~m}$ to the baseboard on one side of the corridor. Mean velocity of the 6 MWT was calculated as walked distance measured by examiner divided over the $6 \mathrm{~min}$. The middle $10 \mathrm{~m}$ track of the corridor was used to apply 10 MWT following the test instructions. At the beginning of the $10 \mathrm{~m}$ track, a 14 -feet long GaitRite was installed. To evaluate the test-retest reliability of temporal and spatial gait parameters calculated by GaitRite and 10 MWT, three measures were carried out with 2 min of separation between them, at first, third and fifth minute of the 6 MWT. Thirty-one temporal and spatial gait parameters calculated based on GaitRite data were selected for analysis as follows: 17 related to velocity, one to frequency, five to time, four to space, seven to gait symmetry and seven to gait stability. Mean value was calculated in some cases, where GaitRite's software measures spatial-temporal parameters for each foot. GaitRite's software calculates symmetry as the absolute mean difference between left and right foot parameter values for step time, step length and duration of gait cycle. ${ }^{23}$ We defined symmetry as symmetry index (SI) for step time, single support, stride velocity, double support, step length, stride length and step angle according to Patterson et al. ${ }^{24}$ using equation 1 as no symmetry equation has demonstrated a clear advantage in terms of distribution or discriminative ability between healthy and pathological subjects. ${ }^{24}$

$$
\mathrm{SI}=\mid(\mathrm{L} \text { value }-\mathrm{R} \text { value }) / 0.5(\mathrm{~L} \text { value }+\mathrm{R} \text { value }) \mid \times 100 \%
$$

(equationl)

where $\mathrm{R}$ stands for right foot and $\mathrm{L}$ means left foot.

\section{Statistical analysis}

As it was stated before, in order to evaluate the test-retest reliability of temporal and spatial gait parameters calculated by GaitRite and 10 MWT, three measures were carried out with $2 \mathrm{~min}$ of separation between them, at first, third and fifth minute of the 6 MWT. Assessment of the 10 MWT and the 6 MWT was carried out in order to reduce measurement bias.

Shapiro-Wilk test was applied to all variables in order to assess normality. Differences along the three tests were calculated by analysis of variance or k-related sample's Friedman test according to variables' normality and homogeneity of variance. ICC was used to assess the test-retest reliability. S.e.m. and SRD were also calculated for each parameter with high ICC; details about this calculation can be found elsewhere. ${ }^{11,25}$

Criteria validity was also evaluated only for GaitRite parameters with excellent test-retest reliability by means of concurrent validity. Concurrent validity of temporal-spatial parameters is demonstrated when GaitRite indicators of gait capacity correlate well with the already validated measures for gait capacity (10 MWT and 6 MWT) and gait performance (6 MWT). To study construct validity, we hypothesize that spatial-temporal GaitRite parameters are highly correlated to $10 \mathrm{MWT}$ and $6 \mathrm{MWT}$ tests, and a lower correlation was seen for LEMS, the use of gait facilitators, SCIM-III total score and SCIM-III items 12,13 and 14 of SCIM-III. Comparison of spatial-temporal GaitRite parameters with gait tests that measure gait capacity (10 MWT and $6 \mathrm{MWT}$ ) was used to study convergent validity. For the discriminant component, 
GaitRite measures were correlated to different constructs such as lower limb muscle capacity (LEMS), global independence (SCIM-III total score), use of gait facilitators and gait performance (SCIM-III items 12, 13 and 14).

To ensure the quality of results of the tests used as gold standards, testreliability of 10 MWT was calculated; also validity of 10 MWT and 6 MWT was established by direct comparison between them and also with WISCI-II and SCIM-III. Validity coefficients $(r)$ were reported using Pearson's productmoment correlation coefficient or Spearman's rank correlation based on distribution of variables. Validity was considered poor if correlation coefficients were between 0.50 and 0.90 and excellent if they were higher than 0.90 .

Statistical analysis was performed in SPSS (version 12.0, Chicago, IL, USA). Results were considered statistically significant if $P<0.05$.

\section{RESULTS}

Twenty-three incomplete SCI subjects AIS D were recruited, with mean age 45.6(12.6) years; 15 were male and eight were female; mean post-injury time was $42(117)$ months, and the majority of them used no aids to walk. Between the subjects who needed one, a cane was the most frequent (see Table 1).

Five spatio-temporal parameters assessed by GaitRite showed statistically significant differences along three tests $(P<0.05)$ difference of step length of both legs, difference of stride length of both legs, difference of step angle of both legs, coefficient of variation of step length and mean step angle of both legs. Five parameters showed low test-retest reliability (ICC < 0.75) - difference of double support of both legs, difference of single support of both legs and difference on stride velocity of both legs, coefficient of variation of left foot length and coefficient of variation of right foot length.

Test-retest reliability (ICC), s.e.m. and SRD reported for $10 \mathrm{MWT}$ in the present study (ICC $=0.91$, s.e.m. $=9 \mathrm{~cm} \mathrm{~s}^{-1}$, SRD $=26 \mathrm{~cm} \mathrm{~s}^{-1}$ ) are within ranges reported in previous studies (s.e.m. $=5-9.1 \mathrm{~cm} \mathrm{~s}^{-1,9,10,26}$ $\left.\mathrm{SRD}=13-25.4 \mathrm{~cm} \mathrm{~s}^{-16,10}\right)$. Test-retest reliability of 21 temporal and spatial gait parameters calculated by GaitRite was also excellent $($ ICC $\geqslant 0.9)$ see Table 2 . However, s.e.m. and SRD of gait velocity assessed by $10 \mathrm{MWT}$ were 2.7 times larger than the ones obtained using GaitRite, which confirms better precision of the last one.

The largest s.e.m. and SRD for gait velocity-related measures are around 3.48 and $9.65 \mathrm{~cm} \mathrm{~s}^{-1}$, respectively. s.e.m. and SRD for functional ambulation profile are around 3.36 points and 9.32 points, respectively. Maximum s.e.m. and SRD for spatial-related gait measures are $1.48 \%$ and $4.10 \%$, respectively. s.e.m. and SRD for

Table $1 \mathrm{SCl}$ subjects characteristics

\begin{tabular}{lc}
\hline Parameters & Values \\
\hline Number of subjects & 23 \\
Age (years) & $45.6(12.6)$ \\
Gender (male/female) & $15 / 8$ \\
Body mass index & $26.2(4.8)$ \\
Post-injury time (months) & $42(117)$ \\
Neurological level & Number of subjects \\
C1-C4 AIS D & 5 \\
C5-C8 AIS D & 1 \\
T1-S5 AIS D & 17 \\
& \\
Walking aids used & \\
None & 9 \\
One cane & 7 \\
Walker & 6 \\
Two canes & 1 \\
\hline
\end{tabular}

Abbreviation: $\mathrm{SCl}$, spinal cord injury. frequency-related gait measures are 3.12 and 8.65 steps per min, respectively.

Correlations for $10 \mathrm{MWT}, 6 \mathrm{MWT}$ and temporal-spatial GaitRite parameters are shown in Table 2. Space-related and velocity-related parameters obtained from GaitRite behaved similarly. Both type of parameters showed an excellent correlation to gait capacity measures (10 MWT and 6 MWT) and a medium correlation to other domains (lower limb muscle capacity, global independence, gait facilitators and gait performance). Slightly lower correlation of cadence with lower limb muscle capacity was obtained. Cadence showed high correlation to gait capacity, medium correlations to gait performance, gait facilitators, global independence and low correlation to lower limb muscle capacity. Time-related gait parameters showed medium correlations to all domains except for lower limb capacity where correlations were poor.

Results for symmetry- and stability-related temporal and spatial GaitRite parameters are more heterogeneous. Most of the symmetryrelated gait parameters showed adequate correlation to all remaining tests, except for the difference of single support of both legs which showed poor correlation with gait capacity tests (10 MWT and $6 \mathrm{MWT})$. To assess construct validity of the symmetry-related parameters, a correlation between the GaitRite symmetry variables and the difference in strength, or asymmetry, between both legs according to LEMs was carried out. Results showed a strong correlation between the asymmetry in force between right and left legs and the difference of single support of both legs $(r=0.632$, $P=0.001)$, and a moderate one with the difference in step time of both legs $(r=0.433, P=0001)$.

Most of the stability-related parameters showed poor correlations to all reference tests, except for the adequate level of correlation found between mean width of base of support or both legs and the use of gait aids.

\section{DISCUSSION}

Subjects assessed can be considered as a representative sample as the majority of subjects included in the present study were male, with a traumatic SCI in thoracic level, which is similar of the population described by the World Health Organization. ${ }^{27}$

Results showed that instrumented evaluation of spatial-temporal gait parameters can offer high reliable measures owing to the fact that only 10 parameters out of 31 showed differences or low reliability along repetitions. Low reliability and differences along tests could be due to subject's deterioration of gait after repetition of the task as a result of a phenomena related to cardiovascular response, premature exhaustion of neuronal activity, ${ }^{28}$ spasticity or gait biomechanics variability. ${ }^{29}$ Another reason could be a calculation issue as a result of a relatively high variability of the parameters intended to be measured. To clarify those observations, a more profound analysis of those parameters is needed. Even so, a sufficient amount of spatial-temporal parameters remains to assess mobility, stability and symmetry of gait.

This study addressed the concurrent component of criteria validity. Concurrent validity of temporal-spatial parameters was demonstrated based on the high correlation of GaitRite indicators with the already validated measures for gait capacity (10 MWT and 6 MWT) and gait performance (WISCI-II and SCIM-III items 12, 13 and 14). Concurrent validity is demonstrated when a test correlates well with a measure that has previously been validated. Preferably, the two tests are for the same construct but very often tests for different constructs, presumably related, are used. For example, criterion validity of the reference tests used to measure gait capacity (10 MWT and 6 MWT) 
Table 2 Test-retest reliability and validity of gait capacity parameters measured by GaitRite without statistically significant differences along three trials

\begin{tabular}{|c|c|c|c|c|c|c|c|c|c|c|}
\hline \multirow[t]{3}{*}{ Parameters } & \multirow[t]{3}{*}{ S.e.m. } & \multirow[t]{3}{*}{$S R D$} & \multirow{3}{*}{$\begin{array}{c}\text { BL muscle } \\
\text { capacity } \\
\\
\text { LEMS }\end{array}$} & \multirow{3}{*}{$\begin{array}{c}\text { Global } \\
\text { independance } \\
\\
\\
\text { SCIM-III } \\
\text { Total }\end{array}$} & \multicolumn{6}{|c|}{ Gait } \\
\hline & & & & & Facilitator & SCIM-III & SCIM-III & & & \\
\hline & & & & & Aids & Item 12 & Item 13 & WISCI-II & $10 M W T$ & $6 M W T$ \\
\hline Gait velocity ${ }^{a}, \mathrm{f}$ & 3.37 & 9.34 & 0.55 & 0.73 & 0.73 & 0.73 & 0.80 & 0.78 & 0.98 & 0.98 \\
\hline Stride velocity of $\mathrm{LL}^{\mathrm{a}, \mathrm{f}}$ & 3.42 & 9.47 & 0.55 & 0.73 & 0.73 & 0.73 & 0.80 & 0.78 & 0.98 & 0.98 \\
\hline Stride velocity of $R L^{a, f}$ & 3.48 & 9.65 & 0.53 & 0.72 & 0.72 & 0.73 & 0.79 & 0.78 & 0.98 & 0.98 \\
\hline Velocity normalized ${ }^{b, f}$ & 0.04 & 0.12 & 0.55 & 0.73 & 0.75 & 0.75 & 0.80 & 0.79 & 0.98 & 0.97 \\
\hline Stride velocity normalized of $L^{b}, f$ & 0.04 & 0.12 & 0.55 & 0.73 & 0.75 & 0.75 & 0.80 & 0.79 & 0.98 & 0.97 \\
\hline Stride velocity normalized of $R L^{b, f}$ & 0.04 & 0.12 & 0.55 & 0.73 & 0.75 & 0.75 & 0.81 & 0.79 & 0.98 & 0.97 \\
\hline \multicolumn{11}{|l|}{ Frequency } \\
\hline \multicolumn{11}{|l|}{ Time } \\
\hline Percentage of double support ${ }^{\mathrm{d}, \mathrm{f}}$ & 1.48 & 4.10 & -0.45 & -0.71 & -0.72 & -0.71 & -0.76 & -0.75 & -0.88 & -0.86 \\
\hline Percentage of single support ${ }^{d, f}$ & 0.88 & 2.44 & 0.43 & 0.69 & 0.70 & 0.68 & 0.75 & 0.70 & 0.89 & 0.86 \\
\hline Step time of $L L^{d, f}$ & 0.04 & 0.12 & -0.41 & -0.74 & -0.74 & -0.75 & -0.82 & -0.80 & -0.83 & -0.82 \\
\hline Step time of $R L^{d, f}$ & 0.04 & 0.12 & -0.46 & -0.68 & -0.72 & -0.81 & -0.80 & -0.87 & -0.80 & -0.77 \\
\hline Mean step time of $B L^{d, f}$ & 0.04 & 0.12 & -0.43 & -0.74 & -0.73 & -0.80 & -0.83 & -0.87 & -0.85 & -0.83 \\
\hline \multicolumn{11}{|l|}{ Space } \\
\hline Step lengthe,f & 1.60 & 4.43 & 0.57 & 0.62 & 0.70 & 0.62 & 0.68 & 0.52 & 0.96 & 0.96 \\
\hline Step length normalized of $L L^{b, f}$ & $<0.01$ & $<0.01$ & 0.62 & 0.57 & 0.67 & 0.61 & 0.69 & 0.64 & 0.91 & 0.89 \\
\hline Step length normalized of $R L^{b, f}$ & 0.03 & 0.09 & 0.55 & 0.56 & 0.66 & 0.52 & 0.65 & 0.58 & 0.87 & 0.89 \\
\hline Mean stride width of $B L^{e, g}$ & 1.51 & 4.19 & -0.37 & -0.08 & -0.06 & -0.02 & -0.04 & 0.10 & -0.29 & -0.20 \\
\hline Mean width of base of support $B L^{b, g}$, & 1.59 & 4.40 & -0.32 & -0.45 & -0.62 & -0.36 & -0.49 & -0.45 & -0.34 & -0.26 \\
\hline
\end{tabular}

Abbreviations: 6 MWT, 6 min walk test; 10 MWT, 10 m walking test; BL, both legs; LEMS, lower extremities motor score; LL, left leg; RL, right leg; SCIM-III, Spinal Cord Independence Measure III; SRD, smallest real difference; WISCI-II, Walking Index for Spinal Cord Injury II.

Correlation of gait capacity parameters (GaitRite) compared with lower limb muscle capacity (LEMS), global independence (SCIM-III Total score), gait assisstive devices, gait performance (WISCI-II, SCIM-III, SCIM-III item 12 and SCIM-III item 13) and gait capacity (10 MWT and 6 MWT). Correlation coefficients are reported using Pearson's product-moment correlation coefficient and

Spearman's rank correlation based on distribution of variables.

Units: (a) centimeters per second, (b) dimensionless, (c) steps per minute, (d) seconds and (e) centimeters. Reliability: (f) ICC $>0.9$, (g) ICC $>0.75$.

has been obtained by comparing those tests with other scales ${ }^{30,31}$ such as Barthel index, ${ }^{32}$ Berg balance scale, oxygen consumption, leg strength, ${ }^{33}$ chair stands, standing balance, ${ }^{34}$ timed up and go test, ${ }^{35}$ WISCI-II ${ }^{36}$ and stair climbing capacity. ${ }^{35}$ The 10 MWT assesses the short-duration walking capacity by measuring the time (in seconds) that a patient spends to walk a 10 -m-long track. It has an excellent test-retest reliability ICC from 0.97 to $0.983,6,37$ s.e.m. from 5 to $7 \mathrm{~cm} \mathrm{~s}^{-1}$ (refs 6,26,38) and SRD from 13 to $25.4 \mathrm{~cm}^{6,10}$ It has an excellent criterion validity with 6 MWT from -0.86 to $-0.95,9,39$ an adequate to excellent construct validity with WISCI-II $(r=-0.37$ to $-0.795), 39,40$ and an adequate one with LEMS $\left(r=-0.4\right.$ to $\left.-0.39^{39}\right)$. The 6 MWT valuates long-duration walking capacity and cardiovascular exercise capacity. It measures the distance (in meters) walked within $6 \mathrm{~min}$. The $6 \mathrm{MWT}$ presents a problem of standardization because track length and shape influences distance measures because of number and complexity of turns required. ${ }^{41}$ Previous studies in SCI have used distances from 50 feet. ${ }^{42}$ Some of them are recognized as validation studies ${ }^{30}$ and do not report the shape of the track or the frequency of turns. ${ }^{6,41}$ It has an adequate to excellent construct validity with WISCI-II $(r=0.36-0.69)^{39,43}$ and an adequate one with LEMS $(r=0.49-0.55) .{ }^{39}$ The WISCI-II quantifies patient's walking performance in an ordinal scale; a score of 0 indicates that a patient cannot stand and walk and the highest score of 20 is assigned if a patient can walk more than $10 \mathrm{~m}$ without walking aids or assistance. ${ }^{9}$ WISCI-II has an excellent test-retest reliability $(\mathrm{ICC}=0.994),{ }^{44}$ and excellent criterion validity with the Functional Independence Measure 
$(r=0.765)^{45}$ and adequate to excellent construct validity with LEMS $(r=0.32-0.88)^{39,43}$ and an excellent one with SCIM $(r=0.97){ }^{44}$

Reference tests used as gold standards were performed with enough quality since test-reliability of 10 MWT was similar to the reported in literature. GaitRite assessment showed almost three times better precision and sensitivity than $10 \mathrm{MWT}$. Nevertheless, $10 \mathrm{MWT}$ requires an improvement in velocity as large as $26 \mathrm{~cm} \mathrm{~s}^{-1}$ in order to detect a real (clinical) change with $95 \%$ confidence. GaitRite can detect changes as small as $9.34 \mathrm{~cm} \mathrm{~s}^{-1}$ of gait velocity, 8.65 steps per minute, 9.32 points of the functional ambulation profile, $4.43 \mathrm{~cm}$ of step length, $4.10 \%$ of single support, $15.09 \%$ difference of single support of both legs and $4.40 \mathrm{~cm}$ of width of base of support. This is an important fact to be considered when planning a research in which a minimal change is expected; for example, if intervention for gait rehabilitation does not reach a change higher than $9.34 \mathrm{~cm} \mathrm{~s}^{-1}$ of velocity, then an improvement is not likely to be detected.

Interestingly, correlations of velocity GaitRite parameters with 10 MWT and with 6 MWT were similar even though they measure slightly different aspects of gait. A possible explanation of the similarity between $10 \mathrm{MWT}$ and $6 \mathrm{MWT}$ correlations could be that for AIS D subacute and chronic incomplete SCI patients, the endurance is not significantly affected.

Overall, velocity-related, space-related, frequency-related and timerelated gait parameters showed medium to high correlations to all reference tests except for lower limb muscle capacity, where medium to poor correlations were observed. Higher gait velocity, step length, cadence, percentage of single support, symmetry and stability are expected in subjects with larger gait capacity, performance and independence which require lower use of gait aids. Conversely, higher lower limb muscle capacity does not ensure by itself higher gait velocity, step length, cadence, duration of single support, symmetry and stability.

Correlation between GaitRite parameters and SCIM-III was higher for gait performance items (item 12,13 and 14) than for the total score due to the fact that the SCIM-III total score includes other dimensions not related to gait in the assessment. Interestingly SCIMIII Item 13: Mobility for Moderate Distances (10-100 m) and item 14 Mobility Outdoors (more than $100 \mathrm{~m}$ ) showed same correlation values because subjects AIS D subacute and chronic incomplete SCI did not report differences performing these tasks.

Space-related parameters of GaitRite showed smaller correlations with SCIM-III and WISCI-II than those of the velocity-related parameters. Also cadence obtained lower correlations with LEMS. As a result, cadence- and space-related parameters are less likely to predict using lower limb muscle capacity, independence or performance scales. This suggests the need to include a space-related and a frequency-related measure in clinical scales. Both types of parameters are strongly related to gait velocity as assessed by $10 \mathrm{MWT}$ and 6 MWT. Therefore, cadence and step length could be assessed using modifications of $10 \mathrm{MWT}$ and $6 \mathrm{MWT}$.

Although gait symmetry has been proposed as an important complementary measure to gait velocity, ${ }^{46}$ there is no commonly accepted standard for the method used to calculate it. ${ }^{24}$ There is only one reported comparison between methods to calculate gait symmetry. It assesses gait symmetry on stroke survivors and concludes that evaluation of gait symmetry should be performed by calculation of ratio of the right and left limb values for swing time, stance time and step length. ${ }^{24}$ Paterson defined symmetry as a measure of the parallels of spatial-temporal gait variables between the lower limbs. ${ }^{24}$ However, no reliability analysis was performed on those measures. As a result, symmetry-related measures need validation. In this paper, we analyzed some of these recommended measures and also few others that were not analyzed in this previous study. We evaluated construct validity of the symmetry-related parameters with difference, or asymmetry, between strength in left and right legs and found moderate and strong correlations. We showed that asymmetry based on step time and single support methods is highly reliable, while methods based on step length are not. Differences could be due to calculation methods and population particularities. Stroke survivor patients can present more severe gait asymmetries that can worsen with age. ${ }^{46}$ Results stress the necessity of inclusion of symmetry-related measures to complement clinical scales.

Stability-related parameters showed poor correlation with all gait reference indicators, except for the adequate correlation between mean width of base of support and use of gait aids. It is logical as gait aids are used to support gait stability. Moderate correlation could be due to the order assigned to walking aids in our scale. Consequently, it implies that it is difficult to make an estimation of gait stability based on reference clinical scales.

Limitations of this study include the limitation of only SCI AIS D subjects. Populations with more severe gait alterations and lower LEMS were not tested. However, it could not be possible if they present shuffle gait which affects GaitRite's capacity to distinct foot strikes, possibly resulting in inaccurate estimates.

\section{CONCLUSIONS}

Instrumented evaluation of spatial-temporal gait parameters like the one performed by GaitRite is a valid and reliable way to measures characteristics of gait capacity (velocity, space, frequency, time, symmetry and stability) in subacute and chronic incomplete SCI AIS D subjects. GaitRite precision on assessment of gait velocity is $\sim 2.7$ times better than clinical tests. Clinical tests cannot address symmetry or stability properties of gait. Subjects' higher gait velocity is related to more independence (SCIM-III), lower use of walking aids (WISCI-II), better strength in lower limbs (LEMS) and better gait's capacity.

Even though SRD has been proposed as a measure of sensitivity to change, it is in fact a complementary to reliability concept. As a result, evaluation of change sensitivity after an intervention should be carried out in future to complete validation.

\section{DATA ARCHIVING}

There were no data to deposit.

\section{CONFLICT OF INTEREST}

The authors declare no conflict of interest.

1 Mehrholz J, Kugler J, Pohl M. Locomotor training for walking after spinal cord injury. Cochrane Database Syst Rev 2012; (11): CD006676.

2 Lyalka VF, Zelenin PV, Karayannidou A, Orlovsky GN, Grillner S, Deliagina TG. Impairment and recovery of postural control in rabbits with spinal cord lesions. J Neurophysiol 2005; 94: 3677-3690.

3 Lee BB, Cripps RA, Fitzharris M, Wing PC. The global map for traumatic spinal cord injury epidemiology: update 2011, global incidence rate. Spinal Cord 2014; 52. 110-116.

4 Wirz M, Bastiaenen C, de Bie R, Dietz V. Effectiveness of automated locomotor training in patients with acute incomplete spinal cord injury: a randomized controlled multicenter trial. BMC Neurol 2011; 11: 60.

5 Amatachaya S, Amatachaya P, Keawsutthi M, Siritaratiwat W. External cues benefit walking ability of ambulatory patients with spinal cord injury. J Spinal Cord Med 2013; 36: 638-644.

6 Lam T, Noonan VK, Eng JJ. A systematic review of functional ambulation outcome measures in spinal cord injury. Spinal Cord 2008; 46: 246-254. 
7 Jackson AB, Carnel CT, Ditunno JF, Read MS, Boninger ML, Schmeler MR et al. Outcome measures meeting outcome measures for gait and ambulation in the spinal cord injury population. J Spinal Cord Med 2008; 31: 487-499.

8 Alexander MS, Anderson KD, Biering-Sorensen F, Blight a R, Brannon R, Bryce TN et al. Outcome measures in spinal cord injury: recent assessments and recommendations for future directions. Spinal Cord 2009; 47: 582-591.

9 van Hedel HJ, Wirz M, Dietz V. Assessing walking ability in subjects with spinal cord injury: validity and reliability of 3 walking tests. Arch Phys Med Rehabil 2005; 86: 190-196.

10 Burns AS, Delparte JJ, Patrick M, Marino RJ, Ditunno JF. The reproducibility and convergent validity of the Walking Index for Spinal Cord Injury (WISCI) in chronic spinal cord injury. Neurorehabil Neural Repair 2011; 25: 149-157.

11 Beckerman H, Roebroeck ME, Lankhorst GJ, Becher JG, Bezemer PD, Verbeek ALM. Smallest real difference, a link between reproducibility and responsiveness. Qual Life Res 2001; 10: 571-578.

12 World Health Organization. International Classification of Functioning, Disability and Health: Children \& Youth Version: ICF-CY. WHO Press: Geneva, Switzerland. 2007.

13 Lord S, Galna B, Verghese J, Coleman S, Burn D, Rochester L. Independent domains of gait in older adults and associated motor and nonmotor attributes: validation of a factor analysis approach. J Gerontol A Biol Sci Med Sci 2013; 68: 820-827.

14 McGough EL, Logsdon RG, Kelly VE, Teri L. Functional mobility limitations and falls in assisted living residents with dementia. J Geriatr Phys Ther 2012; 98195: 1.

15 Montero-Odasso M, Casas A, Hansen KT, Bilski P, Gutmanis I, Wells JL et al. Quantitative gait analysis under dual-task in older people with mild cognitive impairment: a reliability study. J Neuroeng Rehabil 2009; 6: 35.

16 Nelson AJ, Zwick D, Brody S, Doran C, Pulver L, Rooz G et al. The validity of the GaitRite and the Functional Ambulation Performance scoring system in the analysis of Parkinson gait. Neuro Rehabilitation 2002; 17: 255-262.

17 Kalsi-Ryan S, Singh A, Massicotte E, Arnold PM, Brodke DS, Norvell D et al. Ancillary outcome measures for assessment of individuals with cervical spondylotic myelopathy. Spine (Phila Pa 1976) 2013; 38: 111-122.

18 Stokic DS, Horn TS, Ramshur JM, Chow JW. Agreement between temporospatial gait parameters of an electronic walkway and a motion capture system in healthy and chronic stroke populations. Am J Phys Med Rehabil 2009; 88: 437-444.

19 Fox E, Tester N, Phadke C, Nair P, Senesac C, Howland D et al. Ongoing walking rocovery 2 years after locomotor training in a child with severe incomplete spinal cord injury. Phys Ther 2010; 90: 793-802.

20 Gregory CM, Bowden MG, Jayaraman A, Shah P, Behrman A, Kautz SA et al. Resistance training and locomotor recovery after incomplete spinal cord injury: a case series. Spinal Cord 2007; 45: 522-530.

21 Manella C, Backus D. Gait characteristics, range of motion, and spasticity changes in response to massage in a person with incomplete spinal cord injury: case report. Int J Ther Massage Bodywork 2011; 4: 28-39.

22 Winchester P, McColl R, Querry R, Foreman N, Mosby J, Tansey K et al. Changes in supraespinal activation patterns following robotic locomotor therapy in motorincomplete spinal cord injury. Neurorehabil Neural Repair 2005; 19: 313-324.

23 CIR Systems Inc. GAITRite Electronic Walkway Technical Reference (WI-02-15) Rev.L. Sparta, NJ, USA,2013. Available from: http://www.gaitrite.com/downloads/WI-02-15_ Technical Reference L.pdf.

24 Patterson KK, Gage WH, Brooks D, Black SE, Mcllroy WE. Evaluation of gait symmetry after stroke: a comparison of current methods and recommendations for standardization. Gait Posture 2010; 31: 241-246.

25 Schuck $P$, Zwingmann $C$. The 'smallest real difference' as a measure of sensitivity to change: a critical analysis. Int J Rehabil Res 2003; 26: 85-91.
26 Flansbjer U-B, Holmbäck AM, Downham D, Patten C, Lexell J. Reliability of gait performance tests in men and women with hemiparesis after stroke. J Rehabil Med 2005; 37: 75-82.

27 Bickenbach J, Officer A, Shakespeare T, von Groote P International Perspectives on Spinal Cord Injury. World Health Organization: Geneva, Switzerland, 2013Available from www.who.int.

28 Dietz V, Müller R. Degradation of neuronal function following a spinal cord injury: mechanisms and countermeasures. Brain 2004; 127: 2221-2231.

29 Barbeau H, Ladouceur M, Norman KE, Pépin A, Leroux A. Walking after spinal cord injury: evaluation, treatment, and functional recovery. Arch Phys Med Rehabil 1999; 80: 225-235

30 Rehab Measures: 6 Minute Walk Test. Rehabilitation Measures Database. 2010. Available from: http://www.rehabmeasures.org/Lists/RehabMeasures/DispForm.aspx? ID $=895$ (accessed on 10 March 2016)

31 Rehab Measures: 10 Meter Walk Test. Rehabilitation Measures Database 2010. Available from http://www.rehabmeasures.org/Lists/RehabMeasures/PrintView.aspx?ID = 901. (accessed on 10 March 2016).

32 Tyson S, Connell L. The psychometric properties and clinical utility of measures of walking and mobility in neurological conditions: a systematic review. Clin Rehabil 2009; 23: 1018-1033.

33 Patterson SL, Forrester LW, Rodgers MM, Ryan AS, Ivey FM, Sorkin JD et al. Determinants of walking function after stroke: differences by deficit severity. Arch Phys Med Rehabil 2007; 88: 115-119.

34 Harada ND, Chiu V, Stewart AL. Mobility-related function in older adults: assessment with a 6-minute walk test. Arch Phys Med Rehabil 1999; 80: 837-841.

35 Flansbjer U-B, Holmback AM, Downham D, Patten C, Lexell J. Reliability of gait performance tests in men and women with hemiparesis after stroke. J Rehabil Med 2005; 37: 75-82.

36 van Hedel HJ, Wirz M, Dietz V. Assessing walking ability in subjects with spinal cord injury: validity and reliability of 3 walking tests. Arch Phys Med Rehabil 2005; 86: 190-196.

37 Bowden MG, Behrman AL. Step activity monitor : accuracy and test-retest reliability in persons with incomplete spinal cord injury. J Rehabil Res Dev 2014; 44: 1-9.

38 Musselman KE. Clinical significance testing in rehabilitation research: what, why, and how? Phys Ther Rev 2007; 12: 287-296.

39 van Hedel $\mathrm{HJa}$, Wirz M, Curt A. Improving walking assessment in subjects with an incomplete spinal cord injury: responsiveness. Spinal Cord 2006; 44: 352-356.

40 Lemay J-F, Nadeau S. Standing balance assessment in ASIA D paraplegic and tetraplegic participants: concurrent validity of the Berg Balance Scale. Spinal Cord 2010; 48: 245-250.

41 Scivoletto G, Tamburella F, Laurenza L, Foti C, Ditunno JF, Molinari M. Validity and reliability of the 10-m walk test and the 6-min walk test in spinal cord injury patients. Spinal Cord 2011; 49: 736-740.

42 Olmos LE, Freixes O, Gatti Ma, Cozzo Da, Fernandez Sa, Vila CJ et al. Comparison of gait performance on different environmental settings for patients with chronic spinal cord injury. Spinal Cord 2008; 46: 331-334.

43 Ditunno JF, Barbeau H, Dobkin BH, Elashoff R, Harkema S, Marino RJ et al. Validity of the walking scale for spinal cord injury and other domains of function in a multicenter clinical trial. Neurorehabil Neural Repair 2007; 21: 539-550.

44 Morganti B, Scivoletto G, Ditunno P, Ditunno JF, Molinari M. Walking index for spinal cord injury (WISCI): criterion validation. Spinal Cord 2005; 43: 27-33.

45 Ditunno JF, Ditunno PL, Graziani V, Scivoletto G, Bernardi M, Castellano V et al. Walking index for spinal cord injury (WISCI): an international multicenter validity and reliability study. Spinal Cord 2000; 38: 234-243.

46 Patterson KK, Nadkarni NK, Black SE, Mcllroy WE. Gait symmetry and velocity differ in their relationship to age. Gait Posture. England 2012; 35: 590-594. 